\title{
THERMOCAPILLARY ACTUATION OF LIQUIDS USING PATTERNED MICROHEATER ARRAYS
}

\author{
Joseph P. Valentino ${ }^{\mathrm{a}}$, Student Member, IEEE, Anton A. Darhuber ${ }^{\mathrm{b}}$, Sandra M. Troian ${ }^{\mathrm{b}}$, \\ Sigurd Wagner ${ }^{\mathrm{a}}$, Fellow, IEEE \\ Departments of Electrical Engineering ${ }^{a}$ and Chemical Engineering ${ }^{b}$ \\ Microfluidic Engineering \& Research Laboratory, \\ Center for Photonics and Optoelectronic Materials (POEM) \\ Princeton University; NJ 08544, USA \\ Tel.: (609) 258-2961, Fax: (609) 258-0211, e-mail: jvalenti@princeton.edú
}

\begin{abstract}
We demonstrate a microfluidic actuation technique capable of directing nanoliter liquid samples on the surface of a glass substrate through the use of both electronically addressable heater arrays and chemical patterning. Pathways for liquid movement are delineated by the arrangement of microheaters, which also provide the thermocapillary actuating force. The drops are confined by selectively depositing a fluorinated monolayer to the surface, which defines the channel edges. Operating voltages in the range of $2-3 \mathrm{~V}$ were used to move, split, and trap liquids. This fluid transportation technique enables direct access to liquid samples for handling and diagnostic purposes and offers a low power alternative to existing microfluidic systems.
\end{abstract}

\section{INTRODUCTION}

Recent progress in the development of microfluidic analysis systems has the potential to revolutionize the fields of chemical, biological, and material science. By reducing sample size, advantages such as improved response times, reduced cost per analysis, and increased experimental throughput are made possible. In these devices, various fluid actuation techniques have been implemented for liquid flow in closed channels $[1,2,3]$ and on open surfaces $[4,5,6]$. We present a microfluidic actuation technique that utilizes programmable surface temperature distributions in combination with a patterned non-wetting monolayer to control the direction and flow rate of nanoliter volumes of liquids on a free surface [7]. This system takes advantage of the increased role of surface tension in fluids with large surface area to volume ratios, by allowing one to induce flow via the manipulation of liquid-vapor surface energy. Benefits of this fluidic actuation method include low voltage operation and the, ability to handle either polar or nonpolar liquids.

\section{EXPERIMENTS}

Theory. A liquid film which is heated locally at some position $\mathbf{x}$ reduces the surface tension, $\gamma(\mathbf{x})$, at that point and gives rise to a gradient in surface tension across the liquid. A thermocapillary shear stress $\tilde{\tau} \cdot \hat{n}=\nabla \gamma=(\partial \gamma / \partial T) \nabla T$ is induced which pulls the liquid away from the heated region, where $\tilde{\tau}$ is the shear stress, $T$ is temperature, and $\hat{n}$ is the unit normal direction $[8,9,10]$. For a thin flat liquid film, the flow speed is given by $\boldsymbol{v}(\mathbf{x})=h(\mathbf{x}, t) \tilde{\tau} \cdot \hat{n} / 2 \mu(\mathbf{x})$ where $h(\boldsymbol{x}, t)$ is the film thickness, $\mu(\mathbf{x})$ is the local viscosity., and $t$ is time. The flow rate per unit channel width is then $Q(\mathbf{x}, t)=h(\mathbf{x}, t) v(\mathbf{x}, t)$.

Sample Fabrication. A cross-section through one heater plus the leads of the completed device is shown in Fig. 1(a). The sample design is fabricated as follows: organic contaminants are removed from Corning $17370.7 \mathrm{~mm}$ thick glass slides by placing them in a solution of $1: 1$ $\mathrm{H}_{2} \mathrm{SO}_{4}: \mathrm{H}_{2} \mathrm{O}_{2}$ for $30 \mathrm{~min}$. Ti $(50 \mathrm{~nm})$, $\mathrm{Au}(300 \mathrm{~nm})$, and $\mathrm{Cr}(3 \mathrm{~nm})$ are then deposited onto the glass slides via electron beam evaporation, and are patterned into resistive heating elements and contacts using a lift-off procedure.

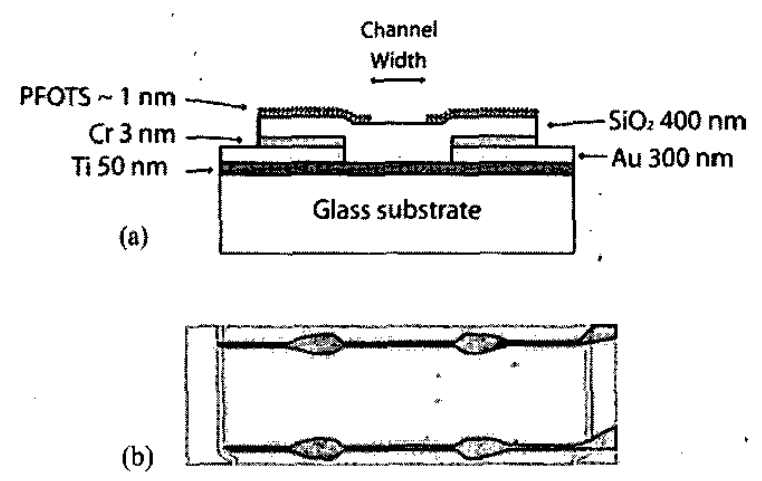

Figure 1. (a) Cross-section of completed thermocapillary actuation device. Maximum power dissipation occurs in the center region of the titanium layer where both $\mathrm{Cr}$ and $\mathrm{Au}$ have been removed. The lower surface energy of the PFOTS monolayer serves to confine liquids over the microheater and defines the width of each droplet. Card edge connectors contact the Au film at both ends. (b) Top-view of a single microheater (heater width $3 \mathrm{~mm}$ ). Constrictions in the heater slightly increase local resistive power dissipation and help center the drops (Note: contact leads are not shown).

Both the $\mathrm{Cr}$ and $\mathrm{Au}$ are removed from the heaters to increase their resistance and therefore concentrate the power dissipation in this area. Constrictions in the heating elements, located below the hydrophilic stripe edges, slightly increase the local resistive heating and help center the moving drops. In order to provide high conductivity leads, the $\mathrm{Cr}$ is etched away in the contact regions regions. With the contact regions masked, a 400 $\mathrm{nm}$ layer of $\mathrm{SiO}_{2}$ is then deposited using plasma enhanced 
chemical vapor deposition at $250^{\circ} \mathrm{C}$ to provide electrical insulation. A monolayer is then formed on the surface by placing the samples in a solution of 1,1,2,2perfluoroctyltrichlorosilane (PFOTS), and is defined using photolithography $[11,12]$. This monolayer establishes non-wetting regions to provide confinement of liquid drops. A top-view of a single microheater is shown in Fig. 1(b). The typical heater size is $0.8 \mathrm{~mm}$ long and 3 mm wide.

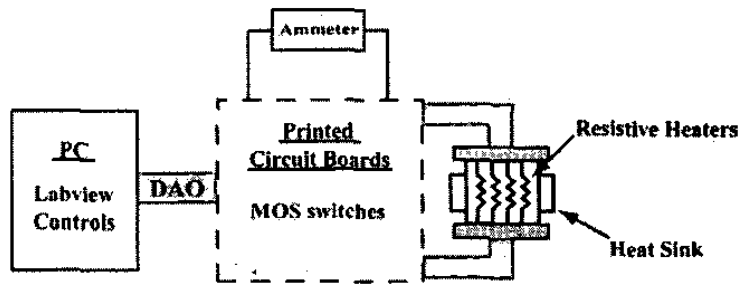

Figure 2. Experimental layout showing the fabricated device on top of heat sink at the right, the switching and measurement electronics in the center, and the computer with the digital-toanalog boards at the left.

Experimental Setup. The experimental setup is shown in Fig. 2. The resistive heaters are addressed via a LabView control program from a PC. The power supply for the heaters comes from two digital to analog output (DAO) boards (United Electronic Industries PD2-AO-32) housed in the PC. The boards provide a total of 64 output channels, each with a maximum allowed voltage and current of $10 \mathrm{~V}$ and $90 \mathrm{~mA}$, respectively. Each voltage supply channel of the DAO is connected to a set of MOSFET switches (Analog Devices ADG452) located on a printed circuit board (PCB). The switches enable each heating resistor to be connected directly to the voltage supply or in series with a Keithley ammeter. By measuring the change in resistance, the ammeter can detect the presence and thickness of a liquid on the surface of the chip [13]. Both sides of the sample are connected to the PCB by card edge connectors with a 1.54 $\mathrm{mm}$ connector spacing. The glass slide is held in place by vacuum on top of a brass block fixed at room temperature. This heat sink reduces lateral heat transfer between adjacent heating elements and also provides a constant operating temperature to prevent the device from heating up continuously.

\section{RESULTS AND DISCUSSION}

The actuation and splitting of discrete dodecane droplets is shown in Fig. 3. The light gray regions correspond to the heating resistors, which are separated by a spacing of $60 \mu \mathrm{m}$, As seen in Fig. 3(a)-3(e), localized heating can split a single droplet drop of approximately $150 \mathrm{~nL}$ of dodecane into two separate drops. Any small satellite drops left behind by the action of splitting (such as the one shown in Fig. 3(e)) can be absorbed by moving one of the larger drops over it. The

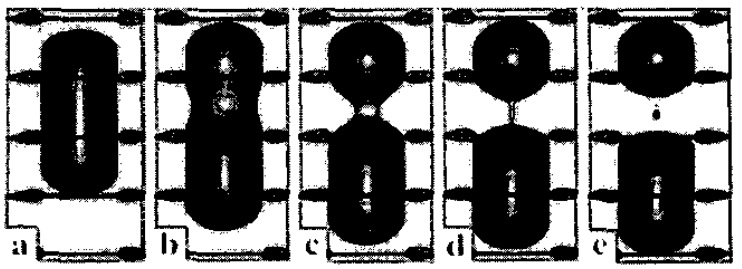

Figure 3. (a)-(e) Thermally induced splitting of a dodecane drop on a partially wetting stripe (droplet width $=1000 \mu \mathrm{m}$ ). Resistive heaters are defined by the light gray regions. The voltage applied to the $155 \Omega$ resistor was $2.5 \mathrm{~V}$. The images were recorded at $t=0,6.0,7.5,8.0$, and $8.5 \mathrm{~s}$.

power used to split and move a drop of dodecane was typically less than $40 \mathrm{~mW}$ per resistor, which provides a thermal gradient of $5^{\circ} \mathrm{C} / \mathrm{mm}$. Thermocapillary actuation of discrete drops is demonstrated in Figs. 4 and 5. Figures 4(a)-4(d) depict a dodecane drop moving from right to left through an intersection of two $1000-\mu \mathrm{m}$ wide partially wetting stripes defined by the monolayer patterning.

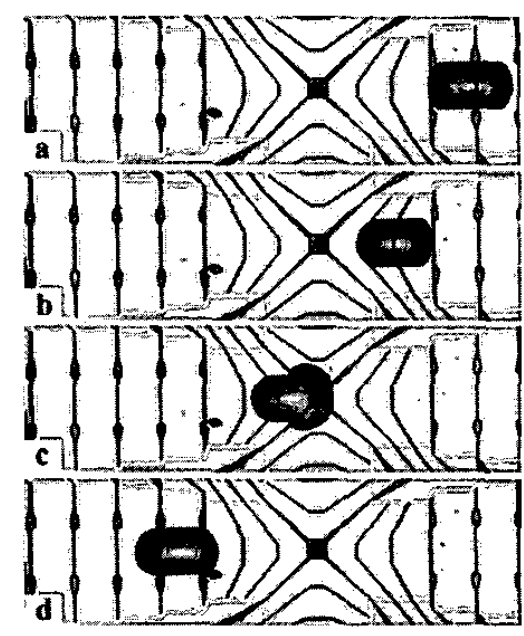

Figure 4. (a)-(d) Thermocapillary actuation of dodecane drop through intersection (droplet width $=1000 \mu \mathrm{m}$, time lapse $=104$ s)

Figures 5(a)-5(c) show another dodecane drop turning a corner at the same intersection. The sequential addressing of resistors is shown in a diagram in Fig. 6. By heating resistors 1 and 3 , the position of the drop is then known to a precision equivalent to the length of one microheater. Resistor 3 is then turned off as resistor 4 is simultaneously turned on. The drop then moves away from its initial position, as it is no longer tightly confined by thermal gradients. Finally, as seen in Fig. 6(c), resistor 2 is turned on and resistor 1 is switched off, and the droplet is now positioned above resistor 3 . The average droplet speed, $v_{d}$, can then be controlled by heater switching times, i.e. varying the time between each step in power. 


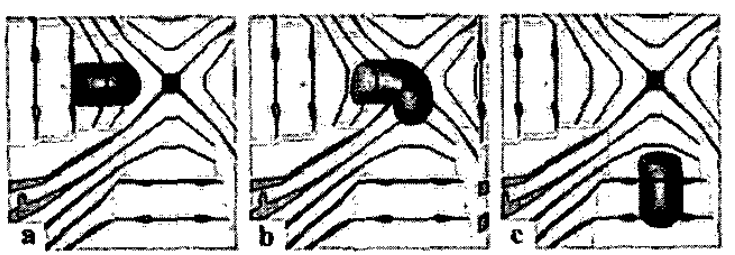

Figure 5. (a)-(c) Dodecane drop turning $90^{\circ}$ corner. Power applied to each resistor $\leq 40 \mathrm{~mW}$ (Time lapse $=164 \mathrm{~s}$ ).

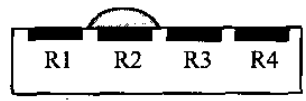

a.) R1 and R3 on

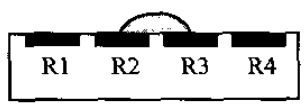

b.) RI and R4 on; R3 off

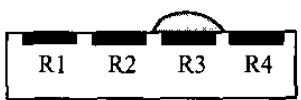

c.) Increase R2 voltage; R4 on; $\mathrm{R} 1$ off

Figure 6. Cross-section of device showing sequential heating of resistors, R1, R2, R3, R4: a.) Voltage applied to R1 and R3: the drop is confined on top of R2. b.) Turn off R3 and apply voltage to R4: drop moves away from R1. c.) Apply voltage to R2 and turn off $\mathrm{R} 1$ : drop is positioned above $\mathrm{R} 3$.

For the droplet in Figs. 4(a)-4(d), $v_{d}=56 \mu \mathrm{m} / \mathrm{s}$ and, $v_{d}=$ $26 \mu \mathrm{m} / \mathrm{s}$ for the droplet in Figs. 5(a)-5(c). A voltage of $2.36 \mathrm{~V}$ was applied to the heaters in these series of images and the heater resistances are $150 \Omega$. On completely wetting surfaces, a thin liquid film can trail behind a moving droplet since the receding contact angle is very small. Glycerol, polyethylene glycols (having molecular weights between 100 and 1000), and polydimethylsiloxane silicone oil have also been transported using thermocapillary actuation, in addition to dodecane. Due to high volatility and contact line pinning, water has not yet provided reliable experimental results. We are experimenting with methods for encapsulation and various surface treatments to reduce these effects. The power required for splitting and transporting liquid drops can be reduced by at least one order of magnitude by using a polymeric substrate of low thermal conductivity instead of glass [13]. Battery operation of this microfluidic device then becomes practicable.

\section{CONCLUSIONS}

We have demonstrated an electronically addressable heating array of microheaters to split, actuate, and direct liquid drops on a free surface. Electronic control of the heating elements allows merging or dividing of liquids into specified volumes, establishing transport with a predetermined velocity, and trapping drops at fixed locations, for eventual analysis and synthesis. These results demonstrate the feasibility of thermocapillary flow as an actuation method for dropletbased microfluidic systems.

\section{Acknowledgements}

This work is funded by NSF grant CTS-0088774, MRSEC grant DMR-9809483, and a NICST grant.

\section{References}

[1] P. Gravesen, J. Branebjerg, and O. S. Jensen, J. Micromech. Microeng. 3, 168 (1993).

[2] T. S. Sammarco and M. A. Bums, AIChE J. 45, 350 (1999).

[3] G. H. W. Sanders and A. Manz, Trends Anal. Chem. 19, 364 $(2000)$.

[4] J. Lee and C. J. Kim, J. Microelectromech. Syst. 9, 171 $(2000)$.

[5] T. B. Jones, M. Gunji, M. Washizu, and M. J. Feldman, $J$. Appl. Phys. 89, 1441 (2001).

[6] Advalytix AG, www.advalytics.com

[7] A. A. Darhuber, J. P. Valentino, J. M. Davis, S. M. Troian, S. Wagner, Appl. Phys. Lett. 82, 657 (2003).

[8] L. G. Leal, Laminar and Convective Transport Processes (Butterworth-Heinemann, Boston, 1992).

[9] V. G. Levich, Physicochemical Hydrodynamics (PrenticeHall, Englewood Cliffs, NJ, 1962).

[10] V. Ludviksson and E.N. Lightfoot, AIChE J. 17, 1166 (1971).

[11] A. A. Darhuber, J. M. Davis, S. M. Troian, and W. Reisner, Phys. Fluids. (submitted).

[12] A. A. Darhuber, S. M. Troian, and W. Reisner, Phys. Rev. E 64, $1063(2001)$.

[13] A. A. Darhuber, S. M. Troian, and S. Wagner, J. Appl. Phys. 91, 5686 (2002). 